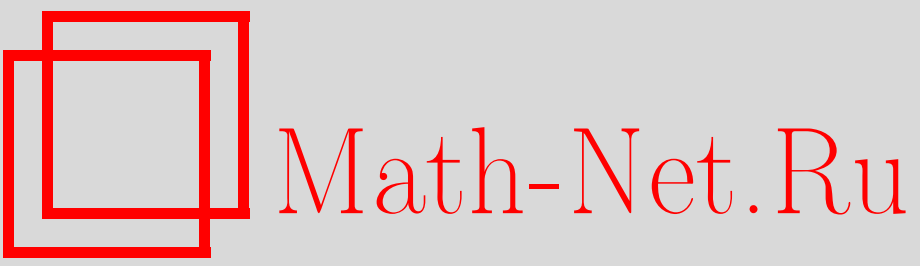

О. И. Богоявленский, Скрытая структура симметрий, УМH, 2004, том 59, выпуск 4, 195-196

DOI: https://doi.org/10.4213/rm764

Использование Общероссийского математического портала Math-Net.Ru подразумевает, что вы прочитали и согласны с пользовательским соглашением

http://www.mathnet.ru/rus/agreement

Параметры загрузки:

IP : 35.173 .219 .149

26 апреля 2023 г., 06:35:32 


\section{СКРЫТАЯ СТРУКТУРА СИММЕТРИЙ}

\section{О. И. БогоявлЕнСкий}

1. В работе представлена скрытая инвариантная флаг-структура в алгебре Ли $A$ симметрий любой динамической системы

$$
\dot{x}^{i}=V^{i}\left(x^{1}, \ldots, x^{N}\right)
$$

на некотором гладком многообразии $M^{N}$. Аналогичные инвариантные фллаг-структуры существуют также в кольцах $R$ первых интегралов, в комплексах $V$-инвариантных дифференциальных форм и в собственных подпространствах любых линейных операторов. Алгебра Ли симметрий системы (1) состоит из векторных полей $U$ на $M^{N}$, коммутирующих с $V:[V, U]=0$, или $L_{V} U=0$, где $L_{V}$ - оператор производной Ли.

Теорема 1. Для любой динамической системы (1) алгебра Ли симметрий А имеет инвариантную флаг-структуру

$$
A \supseteq A_{1} \supseteq A_{2} \supseteq \cdots,
$$

где подалгебры Ли $A_{p}$ являются идеалами в $А$. Имеют место включения

$$
\left[A_{p}, A_{q}\right] \subset A_{p+q} .
$$

Подалгебры Ли $A_{p}$ являются модулями над кольцом $R$ первых интегралов. Кольцо $R$ имеет инвариантную флаг-структуру $R \supseteq R_{1} \supseteq R_{2} \supseteq \cdots$, где все подкольца $R_{p}$ являются идеалами в $R$. Справедливь включения $R_{p} \cdot R_{q} \subset R_{p+q}$. Подалгебры Ли $A_{k}$ (2) определяют дифференцирования колеч $R_{\ell}$, удовлетворяющие соотношениям

$$
A\left(R_{\ell}\right) \subset R_{\ell}, \quad A_{k}(R) \subset R_{k}, \quad A_{k}\left(R_{\ell}\right) \subset R_{k+\ell} .
$$

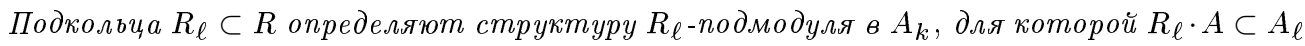
$u R_{\ell} \cdot A_{k} \subset A_{k+\ell}$.

По определению подалгебра Ли $A_{p}$ состоит из симметрий $U_{p} \in A$, имеющих вид $U_{p}=L_{V}^{p} X_{p}$, где $X_{p}$ - некоторое векторное поле на $M^{N}$. Очевидно, $A_{p+1} \subseteq A_{p}$. Подкольцо $R_{p} \subset R$ состоит из первых интегралов системы (1) вида $a_{p}(x)=L_{V}^{p} f_{p}(x)$, где $f_{p}(x)$ - некоторая гладкая функция на $M^{N}$; поэтому $R_{p+1} \subseteq R_{p}$. Для доказательства включений (3) рассмотрим две симметрии $U_{p}=L_{V}^{p} X_{p} \in A_{p}$ и $U_{q}=L_{V}^{q} X_{q} \in A_{q}$ и применим обобщенную формулу Лейбница [1]

$$
L_{V}^{n}\left[X_{p}, X_{q}\right]=\sum_{k=0}^{n} \frac{n !}{k !(n-k) !}\left[L_{V}^{k} X_{p}, L_{V}^{n-k} X_{q}\right] .
$$

При $n=p+q$ формула (5) дает

$$
\left[U_{p}, U_{q}\right]=\frac{p ! q !}{(p+q) !} L_{V}^{p+q}\left[X_{p}, X_{q}\right] \in A_{p+q} .
$$

Включения $R_{p} \cdot R_{q} \subset R_{p+q}$ и (4) следуют из обобщенных формул Лейбница [1] для $L_{V}^{n}(f g)$ и $L_{V}^{n}(X(f))$, где $X$ - векторное поле и $f, g$ - гладкие функции на $M^{N}$.

СлЕДСТвИЕ. Фактор-алгебры Ли $A_{p} / A_{p+q}$ являются нильпотентными при $p, q \geqslant 1 u$ абелевыми при $q \leqslant p$. Если $A_{N}=0$ для некоторого $N$, то все алгебры Ли $A_{1}, \ldots, A_{N-1}$ нильпотентны и при $q \geqslant[(N+1) / 2]$ алгебры Ли $A_{q}$ абелевы. Если алгебра Ли $A_{1}$ содержит некоторую простую алгебру Ли $\mathscr{G}$, то все алгебры Ли $A_{p}$ содержат $\mathscr{G} u$ флаг (2) бесконечен. 
ЗАмечАниЕ. В работе [2] введены мастер-симметрии $Z_{2}$, удовлетворяющие уравнениям $\left[V,\left[V, Z_{2}\right]\right]=0,\left[V, Z_{2}\right] \neq 0$, и векторные поля $Z_{k}$, удовлетворяющие уравнениям $L_{V}^{k} Z_{k}=0$, $L_{V}^{k-1} Z_{k} \neq 0$. Эти векторные поля не принадлежат алгебре Ли симметрий $A$. Их можно рассматривать как обобщенные собственные векторы оператора производной Ли $L_{V}$, удовлетворяющие уравнениям $L_{V}^{k} Z=0$ для всех целшх $k \geqslant 2$.

Пример 1. Покажем, что флаг (2) и флаг $R \supseteq R_{1} \supseteq R_{2} \supseteq \cdots$ могут быть бесконечны. Рассмотрим гамилтонову систему

$$
\dot{p}_{i}=-\frac{\partial H}{\partial q_{i}}, \quad \dot{q}_{i}=\frac{\partial H}{\partial p_{i}}, \quad H=\frac{1}{2} \sum_{j=1}^{n} p_{j}^{2}+\Phi\left(q_{i}\right),
$$

где потенциал $\Phi\left(q_{i}\right)$ - однородная функция степени -2 . Функция $F=p_{1} q_{1}+\cdots+p_{n} q_{n}$, очевидно, удовлетворяет уравнениям $L_{V} F=2 H, L_{V}^{2} F=0$, где $V$ - векторное поле системы (6). Следовательно, гамильтониан $H \in R_{1} \neq 0$ и $H^{k} \in R_{k} \neq 0$. Очевидно, $H^{k} V$ является симметрией системш (6) и $H^{k} V=\left(2^{k} k !\right)^{-1} L_{V}^{k}\left(F^{k} V\right) \in A_{k} \neq 0$ для всех $k \geqslant 1$. Следовательно, флаг (2) бесконечен. Например, это так для интегрируемой системы Калоджеро-Мозера (6) с потенциалом $\Phi\left(q_{k}\right)=\sum_{i \neq j}\left(q_{i}-q_{j}\right)^{-2}$.

ПРЕДЛОЖЕНИЕ. Для любой интегрируемой по Лиувиллю гамильтоновой системь $\dot{p}_{i}=-\partial H / \partial q_{i}, \dot{q}_{i}=\partial H / \partial p_{i}$ с компактными инвариантными подмногообразиями флаг (2) имеет вид $A \supseteq A_{1}, A_{2}=0$. Интегрируемая система тогда и только тогда всюду невырождена по Колмогорову [3] ( в каждой тороидальной области $\mathscr{O}=C_{r} \times \mathbb{T}^{k}$ определенной координатами “действие-угол", где $C_{r}-$ шар радиуса $r$ в координатах $I_{1}, \ldots, I_{k}$ и $\mathbb{T}^{k}-k$-мерный тор с угловыми координатами $\left.\varphi_{1}, \ldots, \varphi_{k}\right)$, когда $A=A_{1}$.

Доказательство использует теорему о симметриях и теорему 10 работы [4].

2. Для любого линейного оператора $L$ в линейном пространстве $B$ над некоторым полем $K$ мы рассмотрим собственные подпространства $B_{\lambda .0}$, отвечающие собственному числу $\lambda: x \in B_{\lambda .0}$, если $L x=\lambda x$. Для любого натуралшного $k$ мы определяем линейные подпространства $B_{\lambda . k}=$ $B_{\lambda .0} \cap(L-\lambda)^{k} B \subseteq B_{\lambda .0}$. Поскольку $(L-\lambda)^{k+1} B \subseteq(L-\lambda)^{k} B$, мш получаем каноническую флаг-структуру в каждом собственном подпространстве $B_{\lambda .0}$ :

$$
B_{\lambda .0} \supseteq B_{\lambda .1} \supseteq B_{\lambda .2} \supseteq \cdots .
$$

Пусть $B_{\lambda_{i}} \supseteq B_{\lambda_{i} .0}$ - пространство обобщенных собственных векторов $x$, удовлетворяющих уравнению $\left(L-\lambda_{i}\right)^{k} x=0$ для некоторого $k$. Пусть $B_{\lambda_{i},-p} \subseteq B_{\lambda_{i}}$ - подпространство, аннулируемое оператором $\left(L-\lambda_{i}\right)^{p+1}$. Очевидно, что $B_{\lambda_{i},-p-1} \supset B_{\lambda_{i} .-p}$. Учитьвая включения (7), получаем флаг-структуру

$$
B_{\lambda_{i}} \supset \cdots \supset B_{\lambda_{i},-1} \supset B_{\lambda_{i} .0} \supseteq B_{\lambda_{i} .1} \supseteq \cdots \supseteq B_{\lambda_{i} . k(i)},
$$

где $B_{\lambda_{i} . k(i)}$ - последнее ненулевое подпространство. Легко проверить, что оператор $\left(L-\lambda_{i}\right)^{p}$ устанавливает изоморфизм

$$
B_{\lambda_{i} . p}=B_{\lambda_{i} .0} \cap\left(L-\lambda_{i}\right)^{p} B_{\lambda_{i}}=B_{\lambda_{i} \cdot-p} / B_{\lambda_{i}-(p-1)} .
$$

ПРИНЦИП СИмМЕТРИИ ДЛЯ ФЛАГА (8). Число различных подпространств $B_{\lambda_{i},-k}$ слева от $B_{\lambda_{i} .0}$ равно числу ненулевых подпространств $B_{\lambda_{i} . k}$ справа от $B_{\lambda_{i} .0}$.

\section{СПИСОК ЛИТЕРАТУРЫ}

[1] J. E. Marsden, T. S. Ratiu. Introduction to Mechanics and Symmetry. New York: Springer-Verlag, 1999. [2] B. Fuchssteiner // Progr. Theoret. Phys. 1983. V. 70. № 6. P. 1508-1522. [3] А. Н. Колмогоров // Докл. АН СССР. 1954. Т. 98. № 4. С. 527-530. [4] О. I. Воgоуavlenskij // Comm. Math. Phys. 1997. V. 184. № 2. P. 301-365.

Математический институт им. В. А. Стеклова РАН, Квинский университет, Кингстон, Канада 